\title{
Bioadhesive drug delivery system of diltiazem hydrochloride for improved bioavailability in cardiac therapy
}

\author{
Yong-Bin Wang ${ }^{1}$, Zhe-Xun Lian ${ }^{1}$, Mei-Na Chen ${ }^{1}$, Lu Zhang ${ }^{1}$, Chang-Yong Zhou ${ }^{1}$ \\ and $\mathrm{Wei} \mathrm{Wei}^{2 *}$ \\ ${ }^{1}$ Department of Emergency Medicine, The Affiliated Hospital of Qingdao University, ${ }^{3}$ Department of Internal Medicine, The \\ People's Hospital of Shinan District, Qingdao, Shandong 266003, China \\ *For correspondence: Email: weiwei6502@gmail.com; Tel/Fax: 0086-531-88382623
}

\begin{abstract}
Purpose: To prepare and evaluate bioadhesive buccal films of diltiazem hydrochloride (a L-type calcium channel blocker) for overcoming the limitations of frequent dosing, low bioavailability and gastrointestinal discomfort of oral delivery.

Methods: Buccal films were prepared by solvent casting technique using sodium carboxymethylcellulose, polyvinyl pyrrolidone K-30 and polyvinyl alcohol. The films were evaluated for weight, thickness, surface $\mathrm{pH}$, swelling index, in vitro residence time, folding endurance, in vitro release, ex-vivo permeation (across porcine buccal mucosa) and drug content uniformity.

Results: The drug content of the formulations was uniform with a range of $18.94 \pm 0.066$ (F2) to $20.08 \pm$ $0.07 \mathrm{mg}$ per unit film (F1). The films exhibited controlled release ranging from $58.76 \pm 1.62$ to $91.45 \pm$ $1.02 \%$ over a period $>6 \mathrm{~h}$. The films containing $20 \mathrm{mg}$ diltiazem hydrochloride, polyvinyl alcohol (10\%) and polyvinyl pyrrolidone $(1 \% \mathrm{w} / \mathrm{v})$ i.e. formulation $\mathrm{F5}$, showed moderate swelling, convenient residence time and promising drug release, and thus can be selected for further development of a buccal film for potential therapeutic uses.

Conclusion: The developed formulation is a potential bioadhesive buccal system for delivering diltiazem directly to systemic circulation, circumventing first-pass metabolism, avoiding gastric discomfort and improving bioavailability at a minimal dose.
\end{abstract}

Keywords: Bioadhesive, Cardiac, Diltiazem, Calcium channel blocker, Buccal film, Bioavailability, Sodium carboxymethylcellulose, Polyvinyl pyrrolidone, Polyvinyl alcohol

Tropical Journal of Pharmaceutical Research is indexed by Science Citation Index (SciSearch), Scopus, International Pharmaceutical Abstract, Chemical Abstracts, Embase, Index Copernicus, EBSCO, African Index Medicus, JournalSeek, Journal Citation Reports/Science Edition, Directory of Open Access Journals (DOAJ), African Journal Online, Bioline International, Open-J-Gate and Pharmacy Abstracts

\section{INTRODUCTION}

In recent years, mucoadhesive drug delivery systems (MDDSs) have been found to be very promising for effective and improved systemic or local drug delivery of variety of drugs. MDDSs deliver the drug through various different mucosa (including oral/buccal, nasal, vaginal or rectal) and hence prevent hepatic first pass metabolism, provide prompt action, reducing gastrointestinal irritation and reducing dosage frequency [1].

The concept of mucoadhesion may be defined as the intimate contact of a biomaterial or mucous membrane and drug delivery device for extended period of time [2,3]. The mucoadhesion is mediated with swelling, branch entanglement between mucosa and MDDS followed by 
diffusion across the mucosa. The buccal mucosa is one of the most suitable sites for MDDS [2,48]. Attempts have been made to formulate various mucoadhesive devices including tablets, films, patches, disks, strips, ointments and gels [9-16]. Buccal film may be preferred over adhesive tablet in terms of flexibility and comfort. In addition, they can circumvent the relatively short residence time of oral gels on the mucosa, which are easily washed away and removed by saliva. Moreover, the buccal films are able to protect the wound surface, thus reducing pain and treating oral diseases more effectively [17].

Diltiazem hydrochloride is a widely used cardiovascular drug, for the treatment of angina, essential hypertension and atrial flutter $[18,19]$. When administered orally, frequent dosing is needed due to short biological half-life $\left(t_{1 / 2}, 4 \mathrm{~h}\right)$. It also undergoes high hepatic first pass metabolism leading to low bioavailability (40 \%). It has also been reported to cause gastrointestinal discomfort. Buccal route of drug administration may be a promising approach to overcome the above problems. Thus the present work deals with the formulation and characterization of mucoadhesive buccal films of diltiazem hydrochloride using mucoadhesive polymers.

\section{EXPERIMENTAL}

\section{Materials}

Diltiazem hydrochloride, polyvinyl pyrrolidone K30 (PVP), polyvinyl alcohol (PVA) and sodium carboxymethylcellulose, 1500-400cps (SCMC) were procured from Sigma Aldrich US. All other reagents used were of analytical grade.

\section{Preparation of bioadhesive buccal films}

Buccal films of diltiazem hydrochloride were prepared by solvent casting technique employing aluminum foil cups (placed on glass surface) as substrate [2]. Composition of a circular cast film of various formulations is mentioned in Table 1.
The mucoadhesive films were prepared using ionic (SCMC), non-ionic (PVA) and water-soluble (PVP) polymers. PVP was added in concentrations of 1 and $5 \% \mathrm{w} / \mathrm{v}$ in the formulations to improve film performance and release characteristics.

For SCMC (3\% w/v), the calculated amount of the polymer was dispersed in three forth volume of water with continuous stirring using mechanical stirrer and the final volume was adjusted with distilled water. In case of PVA films, PVA powder $(10 \% \mathrm{w} / \mathrm{v})$ was dissolved in hot water at approximately $80-100{ }^{\circ} \mathrm{C}$ with stirring. Two percent $\mathrm{w} / \mathrm{v}$ diltiazem was incorporated in the polymeric solutions after levigation with $5 \% \mathrm{v} / \mathrm{v}$ glycerol added as a plasticizer. The medicated gels were left overnight at room temperature to ensure clear, bubble-free gels. The gels were cast into aluminum foil cup ( $4.5 \mathrm{~cm}$ diameter), placed on a glass surface and allowed to dry in a leveled oven maintained at 400 , till a flexible film was formed. The dried films were cut into films of 20 $\mathrm{mm}$ diameter, packed in aluminum foil and stored in a desiccator until further use.

\section{Evaluation of mucoadhesive buccal films}

For evaluation of film weight three films of each formulation were taken and weighed individually in digital balance. The average weights were calculated. Three films of each formulation were taken and the film thickness was measured using Micrometer Screw Gauge (Mitutoyo MMO-25DS) at three different places and the mean value was calculated. For determination of surface $\mathrm{pH}$ three films of each formulation were left to swell for $2 \mathrm{~h}$ on the surface of an agar plate. The surface $\mathrm{pH}$ was measured by means of a $\mathrm{pH}$ paper placed on the surface of the swollen patch. A mean of three readings was recorded. After determination of the original film weight and diameter, the samples were allowed to swell on the surface of agar plate kept in an incubator maintained at 37 ${ }^{\circ} \mathrm{C}$. Increase in the weight or diameter of the films $(n=3)$ was determined at preset time intervals. Swelling (S) was calculated as in Eq 1.

Table 1: Composition of bioadhesive buccal films of diltiazem hydrochloride

\begin{tabular}{lcccccc}
\hline Ingredient (\%w/v) & $\mathbf{F}_{\mathbf{1}}$ & $\mathbf{F}_{\mathbf{2}}$ & $\mathbf{F}_{\mathbf{3}}$ & $\mathbf{F}_{\mathbf{4}}$ & $\mathbf{F}_{\mathbf{5}}$ & $\mathbf{F}_{\mathbf{6}}$ \\
\hline DILTIAZEM & 2 & 2 & 2 & 2 & 2 & 2 \\
$\begin{array}{l}\text { HYDROCHLORIDE } \\
\text { Sodium carboxymethyl- }\end{array}$ & 3 & 3 & 3 & - & - & - \\
cellulose (1500-400cps) & - & - & - & 10 & 10 & 10 \\
Polyvinyl alcohol & 0 & 1 & 5 & 0 & 1 & 5 \\
Polyvinylpyrrolidone K-30 & 5 & 5 & 5 & 5 & 5 & 5 \\
Glycerol (\%v/v) & & & & & & \\
\hline
\end{tabular}


$S(\%)=\{(X t-X o) / X o\} 100$

where $\mathrm{Xt}$ and $\mathrm{Xo}$ are the weights of the swollen film after time $t$ and initial film weight at zero time, respectively.

Folding endurance was determined by repeatedly folding a small strip of film of size $(2 \times$ $2 \mathrm{~cm})$ at the same place till it broke $(n=3)$. The number of times, the film could be folded at the same place without breaking gave the value of folding endurance $[2,3,9]$.

\section{Drug content assay}

Three film units of each formulation were taken in separate $100 \mathrm{ml}$ volumetric flask, added $100 \mathrm{ml}$ of $\mathrm{pH} 6.6$ phosphate buffer and kept for $24 \mathrm{~h}$ under constant stirring. The solutions were filtered, diluted suitably and analyzed at $237 \mathrm{~nm}$ in a UV spectrophotometer $(n=3)$.

\section{Evaluation of in vitro residence time}

The in vitro residence time was determined using IP disintegration apparatus. The disintegration medium was composed of $800 \mathrm{ml} \mathrm{pH} 6.6$ phosphate buffer (PB) maintained at $37 \pm 2{ }^{\circ} \mathrm{C}$. The segments of rat intestinal mucosa, $3 \mathrm{~cm}$ length, were glued to the surface of a glass slab, vertically attached to the apparatus. Three mucoadhesive films of each formulation were hydrated from one surface using pH 6.6 PB and then the hydrated surface was brought into contact with the mucosal membrane.

The glass slab was vertically fixed to the apparatus and allowed to move up and down. The film was completely immersed in the buffer solution at the lowest point and was out at the highest point. The time required for complete erosion or detachment of the film from the mucosal surface were recorded $(n=3)$.

\section{In vitro release studies}

The USP XXIV six station dissolution apparatus type 1 was used throughout the study. One Film of each formulation was fixed to the central shaft using a cyanoacrylate adhesive. The dissolution medium consisted of $900 \mathrm{ml} \mathrm{pH} 6.6$ phosphate buffer (PB). The release study was performed at $37 \pm 0.5^{\circ} \mathrm{C}$ with a rotation speed of $50 \mathrm{rpm}$. The release study was carried out for $6 \mathrm{~h}$. After every one-hour, samples were withdrawn from each station, filtered, diluted suitably and then analyzed spectrophotometrically at $237 \mathrm{~nm}$. The data presented are the mean of three determinations.

\section{Ex-vivo permeation studies}

The animal study protocols were approved by the Animal Ethical Committee of Qingdao University, Qingdao (approval no. 201483222). The studies were carried out in compliance with Directive 2010/63/EU on the handling of animals used for scientific purposes [20]

The ex-vivo permeation studies of mucoadhesive buccal films of diltiazem hydrochloride (through a thick excised layer of porcine buccal mucosa) were studied using the modified Franz diffusion cell. A $2.0 \mathrm{~cm}$ diameter film of each formulation under study was placed in intimate contact with the excised porcine buccal mucosa and the topside was covered with aluminum foil as a backing membrane. Teflon bead was placed in the receptor compartment filled with $25 \mathrm{ml}$ of $\mathrm{pH}$ 7.4 phosphate buffer. The cell contents were stirred with a magnetic stirrer and temperature of $37 \pm 10$ was maintained throughout the experiment. Samples were withdrawn at every one hour, filtered, diluted suitably and then analyzed using UV-Visible spectrophotometer.

\section{Statistical analysis}

Results are expressed as mean \pm standard deviation (SD). Statistical analysis was carried out using analysis of variance (ANOVA) on GraphPad Prism $\odot 4.0$ (Graphpad Software Inc. San Diego, CA, USA). $P<0.05$ was considered statistically significant.

\section{RESULTS}

Mucoadhesive buccal films of diltiazem hydrochloride were prepared using mucoadhesive polymers SCMC, PVP and PVA. The drug delivery system was designed as a matrix. The physical characteristics as well as the bioadhesive performance of various films are given in Table 2.

It was found that film thickness were in the range of $0.49 \pm 0.28$ to $0.99 \pm 0.74 \mathrm{~mm}$ and weight in the range of $116 \pm 1.86$ to $129 \pm 0.77 \mathrm{mg}$. Surface $\mathrm{pH}$ of the films was in the range of $5-6$.

The effect of diltiazem hydrochloride on the swelling behaviour and the residence time of the mucoadhesive polymers were observed (Table 2). The folding endurance was measured manually, films were folded repeatedly till it broke, and it was considered as the end point. Folding endurance were found to be high (470 \pm 5) for F6 and low (189.96 \pm 4.04$)$ for F3. 
Table 2: Physicochemical characteristics of bioadhesive buccal films of diltiazem hydrochloride

\begin{tabular}{lcccccc}
\hline Code & $\begin{array}{c}\text { Film weight } \\
\mathbf{( m g})\end{array}$ & $\begin{array}{c}\text { Thickness } \\
\mathbf{( m m})\end{array}$ & $\begin{array}{c}\text { \% Swelling } \\
\mathbf{( 2 h )}\end{array}$ & $\begin{array}{c}\text { In vitro } \\
\text { residence } \\
\text { time } \mathbf{( h )}\end{array}$ & $\begin{array}{c}\text { Folding } \\
\text { endurance }\end{array}$ & $\begin{array}{c}\text { Drug loading } \\
\mathbf{( m g )}\end{array}$ \\
\hline F1 & $129 \pm 0.771$ & $0.49 \pm 0.28$ & $60.14 \pm 1.35$ & $2.5 \pm 0.559$ & $320.0 \pm 7.81$ & $20.08 \pm 0.07$ \\
F2 & $116 \pm 1.86$ & $0.55 \pm 0.13$ & $79.36 \pm 1.84$ & $2.5 \pm 0.721$ & $279.66 \pm 5.5$ & $18.94 \pm 0.06$ \\
F3 & $119 \pm 2.63$ & $0.52 \pm 0.34$ & $51.85 \pm 2.54$ & $3.0 \pm 0.908$ & $189.96 \pm 4.0$ & $19.14 \pm 0.05$ \\
F4 & $117 \pm 1.74$ & $0.98 \pm 0.36$ & $28.67 \pm 1.42$ & $3.25 \pm 0.087$ & $262.33 \pm 3.5$ & $19.92 \pm 0.01$ \\
F5 & $126 \pm 1.93$ & $0.83 \pm 0.54$ & $26.01 \pm 1.57$ & $3.75 \pm 0.109$ & $209.33 \pm 9.0$ & $19.85 \pm 0.02$ \\
F6 & $122 \pm 2.04$ & $0.99 \pm 0.74$ & $25.41 \pm 1.23$ & $1.25 \pm 0.192$ & $470.0 \pm 5.0$ & $19.59 \pm 0.03$ \\
\hline
\end{tabular}

Drug content in formulations was uniform with a range of $18.94 \pm 0.066$ (F2) to $20.08 \pm 0.07(\mathrm{~F} 1)$. In vitro release studies of various formulations were performed in $\mathrm{pH} 6.6$ phosphate buffer at $237 \mathrm{~nm}$. After $6 \mathrm{~h}$ the release was found to be $91.45,79.89,73.65,31.02,58.76$ and $40.45 \%$ in formulation $F 1, F 2, F 3, F 4, F 5$ and F6, respectively (Figure 1).

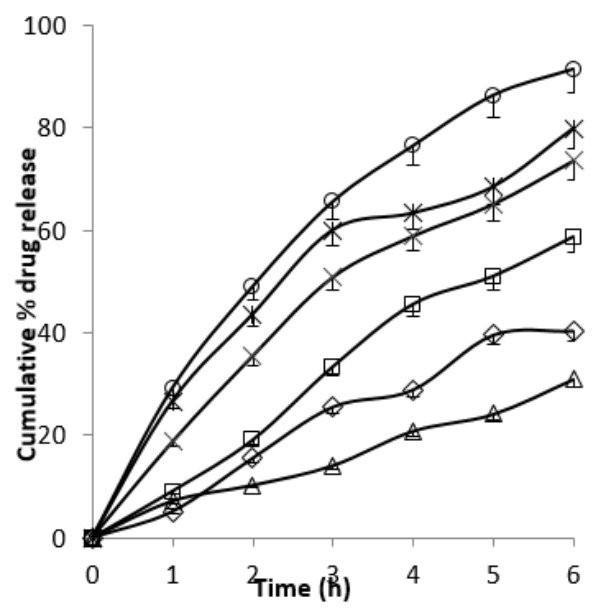

Figure 1: Cumulative percent drug release in $\mathrm{pH} 6.6$ phosphate buffer; Formulation $F_{1}\left({ }^{\circ}\right), F_{2}(*), F_{3}(\times), F_{4}$ $(\Delta), F_{5}(\square), F_{6}(\diamond)$

Among the SCMC films, F1 (SCMC $3 \%$ ) showed the good release. On the other hand, out of the PVA films, release rate was found to be higher for film containing $1 \% \mathrm{w} / \mathrm{v}$ PVP. The plots of log cumulative percent drug retained versus time were found to be linear to the formulations (Figure 2). On the basis of plots it was concluded that the release of diltiazem from the films have obeyed first order kinetics. The correlation coefficient values were found to be -0.9963 , $0.9879,-0.9980,-0.9922,-0.9886,-0.9875$ for F1, F2, F3, F4, F5 and F6, respectively. It shows that data are in good correlation. Negative values of the correlation coefficient indicate negative slope for the plot.

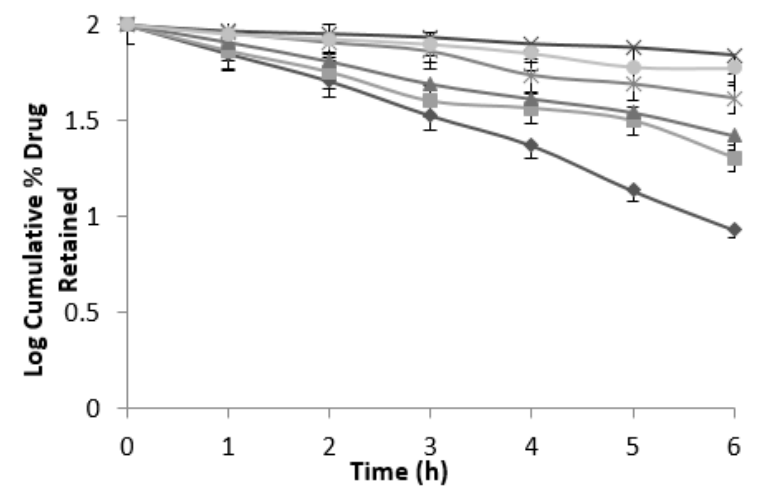

Figure 2: Log cumulative $\%$ drug retained in the formulations. Key: $F_{1}\left({ }^{\circ}\right), F_{2}\left({ }^{*}\right), F_{3}(\times), F_{4}(\Delta), F_{5}(\square)$, $\mathrm{F}_{6}(\diamond)$

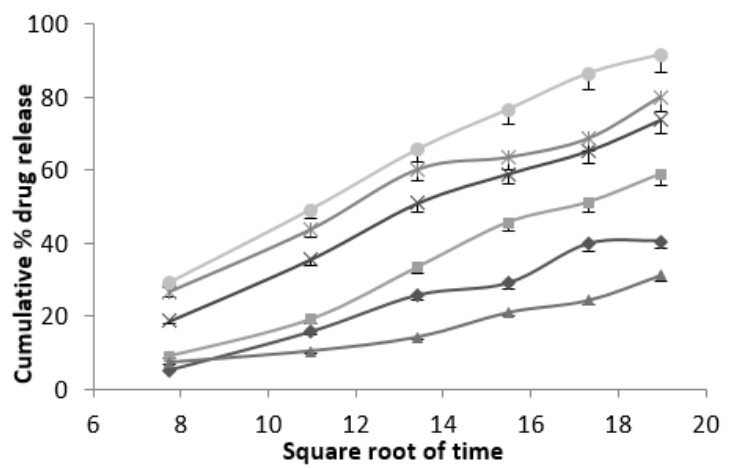

Figure 3: Higuchi plot of the formulations. Key: $F_{1}\left({ }^{\circ}\right)$, $\mathrm{F}_{2}(*), \mathrm{F}_{3}(\times), \mathrm{F}_{4}(\Delta), \mathrm{F}_{5}(\square), \mathrm{F}_{6}(\star)$

Mechanism of drug release pattern i.e. diffusion, swelling or erosion was confirmed by Higuchi plots. Figure 3 shows the graphical representation of cumulative percentage drug release versus square root of time. The Higuchi's Plots were found to be linear with correlation coefficient values of $0.9959,0.9879,0.9980$, 0.9922, 0.9886, 0.9875 for F1, F2, F3, F4, F5 and $\mathrm{F} 6$, respectively. It was concluded that the release of drug from the films followed the diffusion controlled mechanism in all the formulations.

It was also concluded that among the SCMC films formulation $F 1$ showed the promising release pattern as compared to others. From the 
PVA films formulation F5 showed moderate swelling, a convenient residence time as well as adequate drug release. On the basis of release pattern, swelling and residence time F1 and F5 formulations were selected for ex vivo study. In ex vivo study, drug permeation through the porcine buccal mucosa was observed for formulation F1 and F5 (Figure 4).

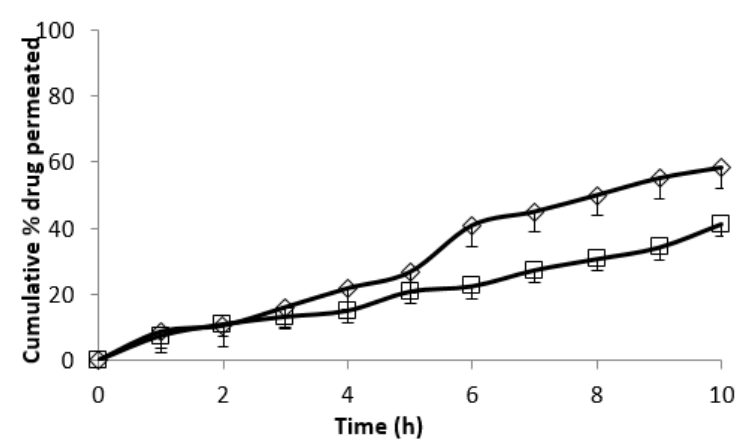

Figure 4: Ex-vivo permeation of diltiazem hydrochloride films in $\mathrm{pH} 7.4$ phosphate buffer.

Key: $F_{1}(\diamond)$ containing SCMC $3 \%$ w/v; $F_{5}$ (口) containing PVA (10\%) and PVP (1 \% w/v) and diltiazem (20 mg)

The drug permeation was found to be 58.25 and $49.01 \%$ in F1 and F5 after $10 \mathrm{~h}$. The Higuchi plots of $\mathrm{F} 1$ and $\mathrm{F} 5$ were almost linear with correlation coefficient values of 0.9310 and 0.9748 , respectively

\section{DISCUSSION}

The medicated films showed high swelling values compared to plain films because of the watersoluble drug, which enhanced the water uptake capacity in the finished dosage form. The influence of drug on the swelling properties of polymer matrices is primarily dependent on the substituted groups of the polymer. The hydroxyl group in the molecules played an important role in the matrix integrity of the swollen hydrophilic cellulose matrices. SCMC containing films showed higher percent swelling as compared to PVA films due to presence of more hydroxyl group in the SCMC molecules. The amount and properties of the incorporated drug determine matrix integrity.

The incorporation of the drug induced significant reduction of the residence time of the studied formulae. The enhanced erosion rate observed with the non-ionic polymers PVA may correlate with the increase in swelling behaviour when the drug was added. As the particle swells, the matrix experiences intra-matrix swelling force promoting disintegration and leaching of the drug leaves behind a highly porous matrix. Water influx weakens the network integrity of the polymer, the structural resistance of the swollen matrices is thus greatly influenced and erosion of the lose gel layer is more pronounced [21]. The early dislodgment of the film from the mucosal surface was more distinct with the ionic polymer SCMC. The addition of PVP predominantly decreased the swelling characteristics of the medicated films of PVA. The water-soluble hydrophilic additive dissolves rapidly introducing porosity. The void volume is thus expected to be occupied by the external solvent diffusing into the film and thereby accelerating the dissolution of the gel [22].

On the basis of drug content determination it was considered that the drug was dispersed uniformly throughout the film. Significant difference was obtained in the release pattern of diltiazem films containing PVA and SCMC. During dissolution SCMC containing films swelled forming a gel layer on the exposed film surfaces. The loosely bound polymer molecules were easily eroded, allowing the release of diltiazem easily as compared to PVA [23]. Both polymers exhibited high swelling, the film weight of these polymers were increased by 25 to $60 \%$ from the initial weight within $2 \mathrm{~h}$ (Table 2). Although the marked increase in surface area during swelling can promote drug release, the increase in diffusion path length of the drug may paradoxically delay the release. In addition, the thick gel layer formed on the swollen film surface is capable of preventing matrix disintegration and controlling additional water penetration [15].

SCMC showed high dissolution rate as compared to PVA. It was found that the drug release from the prepared films varied with respect to the proportion of polymers. Increase in the polymer concentration reduces the diffusion of drug from the matrix. Out of the six formulations, the formulation $F 1$ showed the good release pattern as compared to others and optimum sustained release profile was obtained in formulation F5. So both these formulations only were selected for ex vivo permeation studies. It was observed that the drug permeation followed the matrix diffusion process.

\section{CONCLUSION}

Bioadhesive buccal films of diltiazem have been successfully developed as an effective alternative approach for delivering the drug directly to systemic circulation, and thus, circumventing first pass metabolism, avoiding gastric discomfort and improving bioavailability at minimal dosage. The films, however, require 
further development to make them suitable for therapeutic use.

\section{DECLARATIONS}

\section{Acknowledgement}

The authors acknowledge all those in Qingdao University who directly or indirectly helped with this project.

\section{Conflict of Interest}

No conflict of interest associated with this work.

\section{Contribution of Authors}

The authors declare that this work was done by the authors named in this article and all liabilities pertaining to claims relating to the content of this article will be borne by them.

\section{REFERENCES}

1. Semalty A, Semalty M. Preparation and characterization of mucoadhesive microspheres of ciprofloxacin hydrochloride, Indian Drugs, 2007; 44: 368-372.

2. Semalty $M$, Semalty $A$, Kumar G. Formulation and characterization of mucoadhesive buccal films of glipizide. Indian J Pharm Sci 2008; 70: 43-48.

3. Semalty $A$, Semalty $M$, Nautiyal U. Formulation and evaluation of mucoadhesive buccal films of enalapril maleate, Indian J. Pharm. Sci., 2010; 72: 576-581.

4. Sudhakar Y, Kuotsa K, Bandyopadhyay AK. Buccal bioadhesive drug delivery $-A$ promising option for orally less efficient drugs. J Control Release 2006; 114: 15-40.

5. Streisand JB, Varvel JR, Stanski DR. Absorption and bioavailability of oral transmucosal fentanyl citrate. Anesthesiology. 1991; 75: 223-229.

6. Junginger HE, Hoogstraate JA, Verhoef JC. Recent advances in buccal drug delivery and absorption: in vitro and in vivo studies. J Control Release 1999; 62: 149159.

7. El-Khodairy KA. Effect of physicochemical properties of the hydrophilic Gantrez matrix. Alex J Pharm Sci 2001; 15: 35-40.

8. Jasti $B, L i X$, Cleary $G$. Recent advances in mucoadhesive drug delivery systems. Pharmatech 2003; 194-196.
9. Nafee NA, Ismail FA, Boraie NA, Mortada LM. Mucoadhesive buccal patches of miconazole nitrate: in vitro/in vivo performance and effect of ageing. Int $\mathrm{J}$ Pharm 2003; 264: 1-14.

10. Smart JD. The basics and underlying mechanisms of mucoadhesion. Adv Drug Deliv Rev 2005; 57: 15561568.

11. Ahuja A, Khar RK, Ali J. Mucoadhesive drug delivery systems. Drug Dev Ind Pharm 1997; 23: 489-515.

12. Mazzarino L, Borsali R, Lemos-Senna E. Mucoadhesive films containing chitosan-coated nanoparticles: a new strategy for buccal curcumin release. J Pharm Sci 2014; 103(11): 3764-371.

13. Shojaei A. Buccal mucosa as a route for systemic drug delivery: a review. J. Pharm. Pharm Sci 1998; 1: 15- 30.

14. Khutoryanskiy VV. Advances in mucoadhesion and mucoadhesive polymers. Macromol Biosci. 2011; 11(6): 748-64.

15. Bagan J, Paderni C, Termine N. Mucoadhesive polymers for oral transmucosal drug delivery: a review. Curr Pharm Des 2012; 18(34): 5497-5514.

16. Salamat-Miller N, Chittchang M, Johnston TP. The use of mucoadhesive polymers in buccal drug delivery. Adv Drug Deliv Rev 2005; 57: 1666-1691.

17. Ali J, Khar R, Ahuja A, Kalra R. Buccoadhesive erodible disk for treatment of oro-dental infections: design and characterization. Int J Pharm 2002; 238: 93-103.

18. Sultana $Y$, Mall S, Maurya DP, Kumar D, Das $M$. Preparation and in vitro characterization of diltiazem hydrochloride loaded alginate microspheres. Pharm Dev Technol. 2009; 14(3): 321-331.

19. Das MK, Maurya DP. Evaluation of diltiazem hydrochloride-loaded mucoadhesive microspheres prepared by emulsification-internal gelation technique. Acta Pol Pharm. 2008; 65(2): 249-259.

20. European Commission [homepage on the internet]. Directive 2010/63/EU on the protection of animals used for scientific purposes [cited 2013 December 30]. Available from: http://ec.europa.eu/environment/ chemicals/lab_animals/legislation_en.htm.

21. Wan LSC, Heng PWS, Wong LF. Matrix swelling: a simple model describing extent of swelling of hpmc matrices. Int. J. Pharm. 1995; 116: 168-175.

22. Samuelov $Y$, Donbrow M, Friedman M. Sustained release of drugs from ethyl cellulose-polyethylene glycol films and kinetics of drug release. J Pharm Sci 1979; 68: 325-329.

23. Korsmeyer RW, Gurny R, Doelker E. Mechanisms of solute release from porous hydrophilic polymers. Int $J$ Pharm 1983; 15: 25. 